\title{
Robert LEROUX, Aux fondements de l'industrialisme. Comte, Dunoyer et la pensée libérale en France
}

Jean-Yves Pranchère

\section{(2) OpenEdition}

Édition électronique

URL : http://journals.openedition.org/ress/3574

DOI : $10.4000 /$ ress. 3574

ISBN : $1663-4446$

ISSN : 1663-4446

Éditeur

Librairie Droz

Édition imprimée

Date de publication : 30 novembre 2016

Pagination : 292-294

ISSN : 0048-8046

Référence électronique

Jean-Yves Pranchère, «Robert LEROUX, Aux fondements de l'industrialisme. Comte, Dunoyer et la pensée libérale en France », Revue européenne des sciences sociales [En ligne], 54-2 | 2016, mis en ligne le 22 août 2016, consulté le 25 septembre 2020. URL : http://journals.openedition.org/ress/3574 ; DOI : https://doi.org/10.4000/ress.3574

Ce document a été généré automatiquement le 25 septembre 2020.

(c) Librairie Droz 


\title{
Robert LEROUX, Aux fondements de l'industrialisme. Comte, Dunoyer et la pensée libérale en France
}

\author{
Jean-Yves Pranchère
}

\section{RÉFÉRENCE}

Robert LEROUX, 2015, Aux fondements de l'industrialisme. Comte, Dunoyer et la pensée libérale en France, Paris, Hermann, 168 p.

1 L'objectif affiché par l'ouvrage de Robert Leroux est de «combler une importante lacune » de l'histoire des idées (p. 10). Le but est atteint et on ne peut que se féliciter de disposer d'une présentation synthétique - à la fois érudite et accessible aux nonspécialistes - d'un courant trop oublié du libéralisme français : celui de Charles Comte (1782-1837) et de Charles Dunoyer (1786-1862), qui donnèrent au mot de «libéral » son sens classique en récusant les tendances étatistes de la pensée de Guizot (p. 42-43, p. 123-124, p. 130-133) et en opérant la synthèse des analyses de Jacques Droz, Benjamin Constant, Jean-Baptiste Say et Destutt de Tracy (p. 15-29, p. 51-60, p. 67-72).

Comte et Dunoyer exercèrent une forte influence à travers les journaux Le Censeur et Le Censeur européen qu'ils éditèrent de 1814 à 1819. Ils continuèrent par la suite à fournir des armes doctrinales au libéralisme sous la forme de traités qui enthousiasmèrent Frédéric Bastiat (p. 74, p. 147-149) et furent tenus en haute estime par Auguste Comte (p. 150-152). Leur œuvre, quel que soit le jugement qu'on porte sur elle - anticipation des audaces de l'anarcho-capitalisme (p.40-41) ou témoignage de l'engluement du premier libéralisme dans le régime de notables de la monarchie de Juillet sous laquelle Dunoyer fut préfet (p. 38-39) -, constitue un repère sans lequel l'histoire intellectuelle des années 1815-1848 est difficilement compréhensible.

Sans doute le prestige de la pensée de Saint-Simon a-t-il repoussé dans l'ombre ce libéralisme, «industrialiste » avant la lettre, qui avait accompagné et même précédé 
l'industrialisme saint-simonien. Mais, indépendamment de la question de savoir si Saint-Simon ne se serait pas approprié l'idée de la fondation industrielle des sociétés modernes après l'avoir trouvée chez Comte et Dunoyer (p. 61-64), il convient de ne pas oublier que Saint-Simon avait d'abord été au plus proche des idées du Censeur (auquel participa son secrétaire Augustin Thierry).

4 Il s'en éloigna par la suite et inventa le mot «industrialisme ", en 1824, dans le but explicite d'évincer l'idée de «libéralisme ». Tout en louant les grands mérites de Dunoyer, Saint-Simon lui reprochait d'en rester à une conception purement « critique » du rôle de la classe industrieuse à l'égard du gouvernement. Saint-Simon contestait la thèse libérale selon laquelle le gouvernement a pour unique objet la sécurité des libertés et des propriétés, et la société pour seule mission politique d'obliger le gouvernement à se contenir dans ses plus étroites limites (p. 54, p. 138). Mais SaintSimon ne contestait pas la thèse de Dunoyer selon laquelle «la société devait être constituée pour l'industrie » qui est son «but d'activité » et le moteur du progrès de la civilisation (p.61-63). Il ne faut pas dissimuler ce qu'avait de problématique la dissociation de l'industrialisme et du libéralisme tentée après coup par Saint-Simon, dans une opération théorique qui recevra bien plus tard le nom de "socialisme »: le lien de l'industrialisme et du libéralisme était premier.

Cela ne signifie pas, cependant, que Comte et Dunoyer aient témoigné d'une grande cohérence, plutôt que d'un entêtement dans le déni, en refusant constamment, au nom d'une conception purement négative de la liberté (qui, disait Comte, ne se définit « que par des négations ", p. 88), d'accorder la moindre pertinence à ce qu'on nommait alors la "question sociale». Le simplisme de Comte et de Dunoyer en la matière est confondant : les pauvres sont seuls responsables de leur sort et la paresse est la cause unique de tous les maux sociaux (p. 48-51, p.63, 86, p.111). Ce simplisme - qui conduisit Dunoyer à critiquer les réserves manifestées par Bastiat à l'égard de l'aristocratie anglaise (p. 126-127) - est d'autant plus étonnant que Dunoyer reconnaît que l'inégalité des propriétés, creusée par l'héritage, entrave toujours davantage l'instruction et l'ascension sociale des individus défavorisés (p. 112). Le lecteur est ici fondé à se demander si l'industrialisme de Comte et de Dunoyer n'est pas finalement annulé par un "propriétarisme" qui sacralise la propriété, l'héritage et le libre échange à un tel point qu'il serait prêt à leur sacrifier les conditions sociales et éducatives du «régime industriel» et de l'esprit d'entreprise. Cette tension est perceptible dans le refus opposé par Comte et Dunoyer à tout projet d'éducation publique (p. 46-48, p. 117-119), alors même que les deux publicistes ne cessent d'insister sur le rôle central de la diffusion des connaissances dans la dynamique du progrès industriel (p. 66-67, p. 123).

6 Le choix de Leroux est cependant de ne pas entrer dans ces questions qui relèveraient, soit d'une discussion "normative», soit d'une théorie critique des dialectiques historiques. Adoptant la méthodologie de ses auteurs - celle d'une neutralité scientifique s'en tenant, selon les mots de Comte, à « l'exposition de ce que les choses sont» (p. 77) -, et alors même qu'il reconnaît que ceux-ci ne cessent de tirer des conséquences morales de ce qu'ils donnent pour de simples descriptions factuelles (p. 73, p. 100, p. 117), il s'efforce de laisser parler les penseurs qu'il présente et dont il retrace le parcours sur un mode essentiellement narratif.

7 Sa sympathie pour leur libéralisme n'en est pas moins sensible : il note à plusieurs reprises la «vibrante actualité » des diatribes contre l'inflation des réglementations et 
le poids croissant d'un appareil d'État endetté (p. 18, p. 56, p. 107). Leroux tente même de sauver, dans des pages de Dunoyer (dont il avoue la désuétude) sur l'inégalité des races et des cultures, quelques éléments de vérité (p. 98-99) qui paraîtront bien faibles aux lecteurs de Claude Lévi-Strauss. Mais cette sympathie, qui relève de la «charité interprétative " sans laquelle aucune compréhension n'est possible, n'empiète jamais sur le souci d'objectivité qui le conduit à rendre compte de l'ensemble de la littérature historiographique, y compris lorsque celle-ci consiste en jugements très négatifs, à l'exemple des mots très durs de Stendhal sur la « bêtise » de Dunoyer (p. 63).

Le choix du récit impartial a sa force propre: en terminant son exposé historique par les jugements polémiques de Dunoyer contre la Révolution de 1848 (et en laissant de côté l'attitude de Dunoyer face au Second Empire), Leroux permet de comprendre comment le libéralisme de Dunoyer, indifférent à toute idée d'égalité politique (où il ne voyait qu'un projet d'extension de l'État), centré sur la « liberté du travail » et sur la capacité de la concurrence à produire le lien social (p.116), peut à la fois sembler, par son inégalitarisme, un simple document de la mentalité louis-philipparde et, par son idéal d'une société apolitique où l'État ne fait qu'administrer la sécurité des personnes et des biens, une préfiguration de la pensée libertarienne. Dunoyer définissait le gouvernement comme «une compagnie commerciale, commanditée par la communauté et préposée par elle à la garde de l'ordre public» (p. 108). Le dernier chapitre s'achève par une page de Dunoyer opposant « deux modèles » de république, le français, fondé sur la croissance d'un pouvoir étatique étouffant la liberté sous l'assistanat et la distribution des prébendes, et l'américain, qui laisse «au zèle privé l'initiative de toutes choses» et auquel «des populations intelligentes ne demandent que de la sécurité et de la liberté » (p. 144-145).

Ce n'est cependant par sur cette formulation d'une doxa promise à un riche avenir que se conclut le livre lui-même, mais sur le jugement d'Auguste Comte, qui apercevait dans l'œuvre de Dunoyer une tentative louable, quoique vouée à l'échec, pour élever l'économie libérale à la hauteur d'une sociologie (p. 152). Le livre de Leroux permet de saisir la pleine portée de ce jugement : il montre comment, pour Comte et Dunoyer, l'économie politique, conçue comme "économie de la société » (p.69, p. 114), devait fournit le moyen d'une compréhension de l'auto-organisation de la société industrielle, et donc d'une saisie des lois de cette société en tant que totalité pourvue d'une vie propre (p. 37, p. 58, p. 66-69, p. 94, p. 115) - disons le mot : en tant qu'organisme (p. 104) - et cependant réductible à la psychologie des individus qui la composent (p. 81, p. 120). C'est là le paradoxe le plus profond de l'industrialisme libéral : la tentative de fonder une sorte de sociologie organiciste sur une explication strictement individualiste des relations sociales.

\section{AUTEURS}

\section{JEAN-YVES PRANCHÈRE}

Université libre de Bruxelles, Centre de théorie politique 